\title{
Editorial
}

\section{Calorie Restriction, Longevity and Cognitive Function}

Nazanin Asghari Hanjani, Mohammad Reza Vafa*

Department of Nutrition, School of Public Health, Iran University of Medical Sciences, Tehran, Iran

$\mathrm{O}$ besity is one of the most serious problems of public health and the fifth leading cause of death. It is estimated that $44 \%$ of diabetic load, $23 \%$ of heart ischemic load and $7-41 \%$ of all cancers load are related to obesity. Approximately $80 \%$ of older adults (+65years) have at least one of the above mentioned chronic diseases, and $50 \%$ have at least two chronic disease (1). Along with other organs, brain is also affected with aging and high calorie intake as Fitzpatrik and colleagues suggested in a review article that obese people have problems on decision-making, planning and solving skill, with fewer difficulties on the tasks of verbal fluency, learning and memory (2). In contrast, a reduction of calorie intake without malnutrition called "calorie restriction" (CR) has a wide range of benefits. Moderate CR can prevent or reverse the damaging effects of overweight/obesity, type 2 diabetes, hypertension, chronic inflammation and other age-associated metabolic diseases (1).

As the first groups who worked on CR, Osborne $e t$ al. in 1917 showed that reducing calorie intake in rats increased their length of life. Since then, large amount of time and research efforts have been and continued to be devoted to the study of anti-aging and anti-cancer mechanisms underlying $\mathrm{CR}$ in yeasts, worms, insects, rodents and humans (3). Significant increase in life span has been reported when the nutrient availability drops between 30\% and $75 \%$ of normal calorie provision, according to the species. Not only calorie restricted rodents lived longer than the adlibitum-fed counterparts but a significant part of them (about 30\%) died without any apparent pathology, raising the striking possibility that aging is not necessarily tightly linked with costly pathologies (4).

Preliminary results from human studies are reproducing many of the metabolic and physiological responses sobserved in rodents and monkeys. Short term CR (6-24 months) induces reduction of body weight, subcutaneous and visceral fat, lean body mass, insulin, energy expenditure, and core body temperature (5). Core body temperature, levels of dehydro-epiandrosterone sulfate (DHEAS) and insulin are biomarkers of CR and longevity in human similar to rodents and monkeys. In a 6-month CR related study on overweight individuals, a significant reduction of fasting insulin levels and core body temperature in $\mathrm{CR}$ and $\mathrm{CR}$ with exercise group has been reported; however, DHEAS and fasting glucose remained unchanged, as DHEAS falls 2-4\% per year in humans. The other hypothesis to explain the antiaging effects of $\mathrm{CR}$ is reduced energy expenditure with a consequent reduction in the production of reactive oxygen species (ROS). In this study, a metabolic adaptation over 24 hours following 6 months of CR has also occurred (6). CR decreases serum insulin-like growth factor1 (IGF-1) concentration by approximately $40 \%$ in rodents. This is a key role in protection against cancer and slowing age; as IGF-1 promotes tumor development and inhibits apoptosis (3).

\section{Cognitive impairment and calorie restriction}

Evidence from clinical and basic researches points to a deep connection between brain function decline and metabolic dysregulation during senescence. Excess nutrient availability may be detrimental to brain function. Conversely, a 30\% reduction in 
calorie intake for a period of 3 months was found to improve memory performance in elderly individuals (7). Aside from these observations, the results three ongoing CR studies on rhesus monkeys till now have demonstrated that CR-treated monkeys suffer less severe brain atrophy (a hall mark of aging brain) compared to the control fed adlibitum (8).

Alzheimer's disease: Alzheimer's disease (AD) is a high prevalent neurodegenerative disorder characterized by progressive loss of memory and executive function resulting from synaptic dysfunction and neurodegeneration within vulnerable brain regions (9). Aggregation and accumulation of beta amyloid peptide $(A \beta)$, derived from the amyloid precursor protein (APP) holo-protein, induce synaptic dysfunction, oxidative damage, tau aggregation, and other types of cellular injury that ultimately drive neuropathology associated with the AD disease (10). A study in 1997 showed the scatter plot of AD prevalence versus total food supply for countries; it further indicated that for every per capita additional grams of fat or calorie consumed per day, there is an average increase of $0.03 \%$ and $0.003 \%$, respectively, in $\mathrm{AD}$ prevalence in the adult population of 65 years age or older (7). On the other hand, it has been shown that sustained CR reduces $\mathrm{A} \beta$ neuropathology compared with adlibitum feeding in several AD mouse models (11). Previous studies in which CR started in relatively young mice ( $<7$ months age) with transgenic APP mutations and relatively light amyloid loads demonstrated that CR blocks the mutation of $A \beta$-containing amyloid plaques (12).

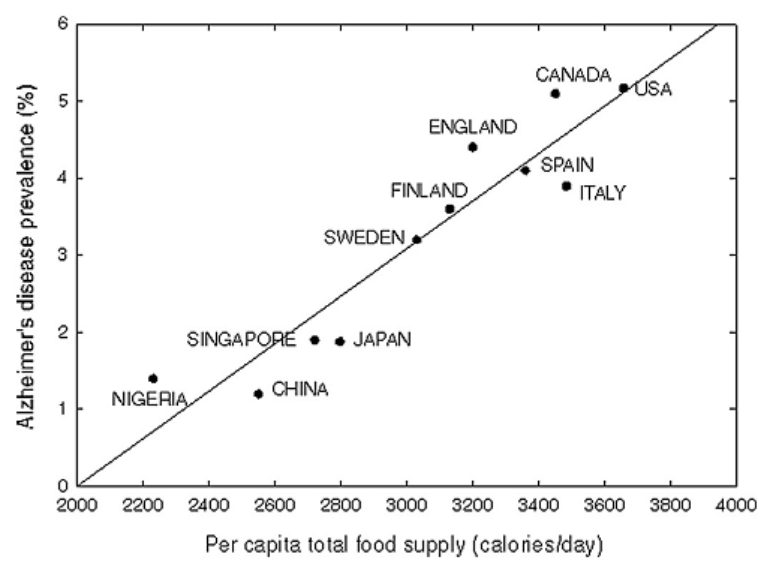

Figure 1. Alzheimer's disease prevalence in +65 years population vs. total food supply.
Parkinson's disease: Parkinson disease (PD) is the second leading age related disorder after $\mathrm{AD}$ with an average onset age of 60 years (13). PD results from the dysfunction and degeneration of dopamine (DA) neurons in the substantia nigra (SN) and DA axon terminals, resulting in progressive motor dysfunction (14). Two findings profoundly influence our science about PD: one mitochondrial dysfunction, oxidative stress and neurodegeneration, and second discovering the genetic cause of PD (15). CR in caenorhabditiselegans whose dopaminergic neurons were degenerated by 6-hydroxyl dopamine showed preventive effect on dopaminergic degeneration by increasing the content of neurotransmitter dopamine (16). Also a study on rhesus monkeys with hemi Parkinson condition treated by $30 \% \mathrm{CR}$ exhibited higher levels of dopamine and dopamine metabolites in stratial pathway compared with the control group. Also it significantly increased the level of dopamine neurons and glial cell line-derived neurotrophic factor, which is known to promote the survival of DA neurons (17).

Huntington: Huntington's disease (HD) is a fatal neurodegenerative disorder caused by an expanded polyglutamine repeat in Huntington protein (18); it is an inherited neurodegenerative disorder with a distinct phenotype, including dystonia, incoordination, cognitive decline, and behavioral difficulties (19). Several studies have suggested that excite toxicity and mitochondrial dysfunction play roles in the pathogenesis of HD (18). It is suggested that higher levels of cellular energy may have a role on disease progression. Because administration of creatine, a ATP-depletion delays the disease onset, and improves neuronal survival (20). CR in Huntington mutant mice increased the level of Brainderived neurotrophic factor (BDNF) and the protein chaperone heat-shock-protein-70 in the stratum and cortex, which were depleted in HD mice fed a normal diet.CR promotes neuronal degeneration by impairing cellular resistance (21).

Underlying mechanisms: There is ample evidence that the mechanisms involve significant alterations in energy metabolism; response to oxidative damage, insulin sensitivity, inflammation, and changes in the neuroendocrine, paracrine and reproductive systems (22) .CR induces a down-regulation of 
inflammatory pathways (e.g., nuclear factor kB), and an up-regulation of genes that enhance protection against molecular damage (e.g., Nrf2 (nuclear factor-erythroid 2), heat shock protein70 (HSP70)) (23). Similar to other organ systems, cells in the brain encounter a cumulative burden of oxidative and metabolic stress that may be universal feature of the aging process and neurodegenerative disorders. Understanding the molecular mechanisms of CR may lead to the development of novel therapies to combat the diseases of aging and to improve the quality of life.

Sirtuins: Sirtuin1(SIRT1) is a (NAD)-dependent deacetylase whose function has been shown to correlate with metabolism, gene transcription, cell division, cellular stress response, and increased life span (12). Indeed, SIRT1 is necessary for increased activity and longevity of animals subjected to CR. In $\mathrm{AD}$, SIRTUN can deacetylate retinoic receptor $\beta$; this in turn results in encoding $\alpha$-secretase, which can reduce the formation of toxic $A \beta$. Increasing level of heat shock protein due to higher amount of SIRT1 can have protective role in PD, and eventually SIRT1 in HD activates the target of rapamysin complex1(TORC1) that interacts with cAMPresponsive element binding protein (CREB), resulting in higher levels of BDNF, a neuroprotective factor in HD (22).

BDNF: Neurotrophins are critical for development and maintenance of the mammalian central nervous system. Among them is brain-derived neurotrophic factor (BDNF); its production is induced by CR. It has a central role in neuronal survival, differentiation and synaptic plasticity, which is important for learning (24). Hipocampal level may be positively related to learning and memory efficiency. It is also negatively correlated to oxidative stress. Furthermore, BDNF has a role in regulating glucose metabolism in peripheral tissues and increase insulin sensitivity (25).

Insulin function: There is a huge burden of research showing that long-term $\mathrm{CR}$ in lean and obese subjects improves insulin sensitivity, a mechanism by which $\mathrm{CR}$ may act to extend lifespan. Additionally, prolonged $\mathrm{CR}$ reduces fasting glucose and insulin concentration, two factors believed to contribute to the aging process due to protein glycation (26). Adipose tissue, which is specifically reduced upon $\mathrm{CR}$ or intermittent fasting regimens, can act as an endocrine organ. It produces trophic hormones, e.g. tumor necrosis factor- $\alpha(\mathrm{TNF} \alpha)$. $\mathrm{TNF} \alpha$ has also been shown to trigger insulin resistance in animals. In vitro cell-culture studies have shown that TNF $\alpha$ renders the cells to be insulin-resistant through a down-regulation of glucose transporter synthesis and also through interference with insulin receptor signaling pathways (27). aging -related cognitive impairment in rats is associated with down-regulation of insulin signaling and pathways that use glucose (28).

In conclusion, $\mathrm{CR}$ in the form of reduced calorie intake manifests protection in all cell tissues and organs including the brain. It retards aging process from the simplest model organisms to humans. CR modulates energy and oxygen radical metabolism, insulin sensitivity, inflammation and cellular stress response systems in a way that protects the cells against the damaging effect of aging and of accumulation higher metabolism end-products due to higher calorie intake that can directly influence on cell cycle, and therefore, disrupting the function of tissues and organs. Since aging is associated with serious chronic diseases, and the population of older people has had an increasing trend during the last century, finding the most effective nutritional way to have a healthy aging with fewer chronic diseases and disabling difficulties can improve the public's health status and quality of life.

\section{Financial disclosure}

The authors declare no financial interest.

\section{Funding/Support}

The study did not receive any financial support.

\section{References}

1. Omodei D, Fontana L. Calorie restriction and prevention of age-associated chronic disease. FEBS 2011; 585(11):1537-42

2. Fitzpatrick S, Gilbert S, Serpell L. Systematic review: are overweight and obese individuals impaired on behavioural tasksof executive functioning? Neuropsychology Review. 2013;23(2):138-56.

3. Fontana L, Weiss EP, Villareal DT, Klein S, Holloszy JO. Long-term effects of calorie or protein restriction on serum IGF-1 and IGFBP-3 concentration in humans. Aging cell. 2008; 7(5.- 681):

4. Taormina G, Mirisola MG. Calorie restriction in mammals and simple model organisms. BioMed Research International. 2014;2014. 
5. Smith DL, Nagy TR, Allison DB. Calorie restriction: what recent results suggest for the future of ageing research. European Journal of Clinical Investigation. 2010;40(5):440-50.

6. Heilbronn LK, de Jonge L, Frisard MI, DeLany JP, Larson-Meyer DE, Rood J, et al. Effect of 6-month calorie restriction on biomarkers of longevity, metabolic adaptation, and oxidative stressin overweight individuals: a randomized controlled trial. JAMA. 2006;295(13):1539-48.

7. Attuquayefio T, Stevenson RJ. A systematic review of longer-term dietary interventions on human cognitive function: Emerging patterns and future directions. Appetite.70_95:554;2015.

8. Pani G, editor Neuroprotective effects of dietary restriction: Evidence and mechanisms. Seminars in cell \& developmental biology; Elsevier. 2015;20:106-114.

9. Almeida CG, Tampellini D, Takahashi RH, Greengard P, Lin MT, Snyder EM, et al. Beta-amyloid accumulation in APP mutant neurons reduces PSD-95 and GluR1 in synapses. Neurobiology of Disease. 2005;20(2):187-98.

10. Schafer MJ, Alldred MJ, Lee SH, Calhoun ME, Petkova E, Mathews PM, et al. Reduction of $\beta$-amyloid and $\gamma$ secretase by calorie restriction in female $\mathrm{Tg} 2576$ mice. Neurobiology of Aging. 2015;36(3):1293-302.

11. Mouton PR, Chachich ME, Quigley C, Spangler E, Ingram DK. Caloric restriction attenuates amyloid deposition in middle-aged dtg APP/PS1 mice. Neuroscience letters. 2009;4643-(7-184;).

12. Ran M, Li Z, Yang L, Tong L, Zhang L, Dong H. Calorie restriction attenuates cerebral ischemic injury via increasing SIRT1 synthesis in the rat. Brain Research. 2015;1610:61-8.

13. Alves G, Forsaa EB, Pedersen KF, Gjerstad MD, Larsen JP. Epidemiology of Parkinson's disease. Journal of Neurology. 2008;255(5):18-32.

14.Blandini F. Neural and immune mechanisms in the pathogenesis of Parkinson's disease. Journal of Neuroimmune Pharmacology. 2013;8(1):189-201.

15.Dauer W, Przedborski S. Parkinson's disease: mechanisms and models. Neuron. 2003;39(6):889-909.

16. Jadiya P, Chatterjee M, Sammi SR, Kaur S, Palit G, Nazir A. Sir-2.1 modulates 'calorie-restrictionmediated'prevention of neurodegeneration in Caenorhabditis elegans: implications for Parkinson's disease. Biochemical and Biophysical Research Communications. 2011;413(2):306-10.

17. Maswood N, Young J, Tilmont E, Zhang Z, Gash DM, Gerhardt GA, et al. Caloric restriction increases neurotrophic factor levels and attenuates neurochemical and behavioral deficits in a primate model of Parkinson's disease. Proceedings of the National Academy of Sciences. 2004;101(52):18171-6.

18. Schilling G, Coonfield ML, Ross CA, Borchelt DR. Coenzyme Q10 and remacemide hydrochloride ameliorate motor deficits ina Huntington's disease transgenic mouse model. Neuroscience Letters. 2001;315(3):149-53.

19. Walker FO. Huntington's disease. The Lancet. 2007;369(9557):218-28.

20. Andreassen OA, Dedeoglu A, Ferrante RJ, Jenkins BG, Ferrante KL, Thomas M, et al. Creatineincreases survival and delays motor symptoms in a transgenic animal model of Huntington's disease. Neurobiology of Disease. 2001;8(3):479-91.

21.Duan W, Guo Z, Jiang H, Ware M, Li X-J, Mattson MP. Dietary restriction normalizes glucose metabolism and BDNF levels, slows disease progression, and increases survival in huntingtin mutant mice. Proceedings of the National Academy of Sciences. 2003;100(5):2911-6.

22.Libert S, Guarente L. Metabolic and neuropsychiatric effects of calorie restriction and sirtuins. Annual review of physiology. 2013;75:669.

23.Omodei D. Calorie Restriction and Anorexia Nervosa: Molecular markers and pathogenetic mechanisms. 2015.

24.Gomez-Pinilla F. The influences of diet and exercise on mental health through hormesis. Ageing research reviews. 2008;7(1):49-62.

25.Kaptan Z, Akgün-Dar K, Kapucu A, Dedeakayoğulları H, Batu Ş, Üzüm G. Long term consequences on spatial learning-memory of low-calorie diet during adolescence in female rats; hippocampal and prefrontal cortex BDNF level, expression of NeuN and cell proliferation in dentate gyrus. Brain research. 2015;1618:194-204.

26.Redman LM, Ravussin E. Caloric restriction in humans: impact on physiological, psychological, and behavioral outcomes. Antioxidants \& redox signaling. 20.87275:(2)14;11

27.Martin B, Mattson MP, Maudsley S. Caloric restriction and intermittent fasting: two potential diets for successful brain aging. Ageing research reviews. 2006;5(3):332-53.

28.Kapogiannis D, Mattson MP. Disrupted energy metabolism andneuronal circuit dysfunction in cognitive impairment and Alzheimer's disease. The Lancet Neurology. 2011;10(2):187-98. 